\title{
Media Manipulations and the Culture of Beneficial Fungal Root Endophytes
}

\author{
Brian R Murphy ${ }^{1}$, Sven P Batke ${ }^{1}$, Fiona M Doohan ${ }^{2} \&$ Trevor R Hodkinson ${ }^{1}$ \\ ${ }^{1}$ Department of Botany, School of Natural Sciences, Trinity College Dublin, Ireland \\ ${ }^{2}$ UCD Earth Institute and School of Biology \& Environmental Science, University College Dublin, Ireland \\ Correspondence: Brian R Murphy, Department of Botany, School of Natural Sciences, Trinity College Dublin, \\ Ireland. E-mail: murphb16@tcd.ie
}

Received: April 17, 2015 Accepted: May 20, 2014 Online Published: May 31, 2015

doi:10.5539/ijb.v7n3p94 URL: http://dx.doi.org/10.5539/ijb.v7n3p94

\begin{abstract}
Fungal endophytes have great potential as biocontrol and biofertilisation agents for food crops, and it is important to understand and optimise the cultural conditions for endophyte emergence and growth. A range of commercial culture media were tested for their effect on endophyte recovery from the roots of their host (Hordeum murinum), and for their subsequent growth and sporulation. Furthermore, a medium composed of sterile or filtered whole plant extract (WPE) was tested at various dilutions both alone and in combination with one of the commercial media. It was found that there were significant differences between the media in endophyte recovery, endophyte mycelial growth and time to sporulation. A significantly greater number of different endophytes were recovered from roots on the malt extract plus WPE medium than any of the other media tested here. Sabouraud medium gave the greatest mean radial growth of endophyte fungal mycelia after 35 days. The cultures growing on the WPE media took over five times longer to sporulate than all other media. Results indicate that different media are suitable either for the initial recovery and isolation of fungal root endophytes or for increasing fungal biomass and inducing earlier sporulation. The experimental endophytes used here have previously been shown to have biocontrol and biofertilisation benefits for barley cultivars, so our findings have important implications for the development and commercialisation of endophyte inocula.
\end{abstract}

Keywords: Hordeum murinum, fungal root endophytes, growth media, plant extract, biofertilisation, biocontrol

\section{Introduction}

Sustainable alternatives to large agrichemical inputs are needed, and endophytes may provide part of the solution. Endophytes are microorganisms (bacteria, fungi and unicellular eukaryotes) which can live at least part of their life cycle inter- or intracellularly inside of plants usually without inducing pathogenic symptoms. This can include competent, facultative, obligate and opportunistic endophytes. Endophytes can have several functions and these may change function during their lifecycle (Murphy, Doohan, \& Hodkinson, 2014). Fungal root endophytes (hereafter 'endophytes') isolated from both unrelated plants (Stein, Molitor, Kogel, \& Waller, 2008; Waller et al., 2005; Achatz et al., 2010; Murphy, Doohan, \& Hodkinson, 2014b) and congeneric species (Murphy, Doohan, \& Hodkinson, 2014c; Murphy, Doohan, \& Hodkinson, 2015) have been shown to have yield and biocontrol benefits for cultivated barley (Hordeum vulgare) and other crop species. In order to fully utilise these organisms it is important to be able to culture the endophytes in an economically effective way and in large enough quantities while maintaining endophyte competence. Many more beneficial endophytes are likely to be discovered in the future and efficient cultural methods are required for their study and will be a major determinant of the commercial potential of these bioproducts. The benefits derived from the use of endophytes are being increasingly investigated, and many are being developed as crop inoculants and for other biotechnological applications (Singh et al., 2003; Behie \& Bidochka, 2013; Kusari et al., 2014).

While the study of fungal growth characteristics on different media has focussed on medical microbiological applications (Meletiadis, Meis, Mouton, \& Verweij, 2001), the cultural preferences of endophytes has received less attention. Research into the optimal growth media and environments for endophyte isolation and culture could be an important contribution to realising their maximum growth and production potential. 
The use of whole plant extract (WPE) as a culture medium for fungi has not been extensively studied, but at least one study has shown increased colony growth of rhizospheric micro-organisms for plant juice-based media over reference nutrient media (Nour et al., 2012). Osman et al. (2013) also demonstrated that WPE from several plant species can act as growth promoters for some fungi. Other studies using WPE have focused on the anti-fungal activity of plant extracts rather than the assessment of effects on mycelial culture and growth (Mohana \& Raveesha, 2007; Dellavalle et al., 2011; Minz, 2012; Yenn et al., 2012; El-Elimat et al., 2014). Artificial growth media are normally used to culture fungal organisms, and different classes of organism grow optimally on different media and under different conditions (Meletiadis et al., 2001). However, the artificial growth media available commercially are nothing like the environment the endophyte would normally experience in the host (Kusari et al., 2014), so by experimenting with available commercial media and plant extracts, we aimed here to discover an ideal medium for the isolation and culture of a particular group of endophytes.

It is important to determine which culture media will perform best for initial isolation and growth of endophytes. It is also essential to establish how existing isolates (maintained in vitro) will grow on differing media, including WPEs or dilutions of these. The in vitro environment is very different to the plant apoplastic environment, and adding WPE from the host species may go some way to optimising culture conditions because of the closer chemical similarity to the host environment. Results obtained using the media containing host plant extract may also indicate potential endophyte growth within barley root tissue, as these media would be closer to the normal endophyte environment. Some commercial production methods for endophyte-based products have used a liquid medium for culture growth, and this type of medium is well-studied (Kumar et al., 2011, Knob et al., 2013). Therefore, we focussed instead on semi-solid agar-based media and sought to determine which of the different media were best for endophyte emergence, growth, proliferation and sporulation.

\section{Materials and Methods}

\subsection{Preparation of Culture Media}

Five commercially available media were used for the isolation of endophytes: corn meal agar (Oxoid CM0103); Czapek Dox liquid agar medium modified (Oxoid CM0095), to which was added $16 \mathrm{~g} / \mathrm{L}$ of Sigma Agar select A-5054 38954; malt extract agar modified, Vegitone (Fluka 38954); potato dextrose agar (Applichem A5838,0500) and Sabouraud maltose agar (Oxoid CM0541). These particular media were chosen as they represent a broad range of different combinations of chemical constituents - varying in nitrogen and carbon sources, $\mathrm{pH}$, and other additives (Table 1). All of these media were prepared at half-strength of the manufacturers' recommendations to prevent osmotic shock in emergent endophytes. Additionally, two media derived from whole plants of wall barley (Hordeum murinum subsp. murinum L.) were used. These media, based on WPE of wall barley (harvested at Zadoks growth stage 07 (Zadoks, Chang, \& Konzak, 1974)) were prepared following a modification to the protocol of Nour et al. (2012). Briefly, whole wall barley seedlings were collected from the field in early summer before flowering shoot emergence and surface-sterilized by washing soil residues from the roots using ultra-pure water, soaking in $5 \% \mathrm{NaClO}$ for 15 minutes, then rinsing three times in ultra-pure water. Seedlings were air dried in a laminar flow cabinet and $40 \mathrm{~g}$ of the sterilised seedlings were blended in a kitchen blender for 5 min with 1:1 w/v ultra-pure water to prepare the base extract. Two dilutions of WPE were prepared in ultra-pure water (WPE 1:2 or WPE 1:30). These dilutions were chosen to test whether different concentrations of WPE produced different effects. Twelve grams of agarose gel powder were added to one litre of WPE dilutions, and the mixture was boiled and then sterilised by autoclaving.

Table 1. Nutritional and additive profiles for each medium, amounts are per culture dish

\begin{tabular}{|c|c|c|c|c|c|}
\hline \multirow[t]{2}{*}{ Medium } & \multirow[t]{2}{*}{ pH } & \multicolumn{4}{|c|}{ Nutritional profile and additives, as prepared } \\
\hline & & $\begin{array}{l}\text { Carbon source / } \\
\text { amount g }\end{array}$ & $\begin{array}{l}\text { Nitrogen source / } \\
\text { amount g }\end{array}$ & Agar content $g$ & Other additives / amount g \\
\hline Corn meal extract & 6.0 & Sugars / 0.04 & $\mathrm{~N}$ content / 0.003 & 0.1 & \\
\hline Czapek Dox & 6.8 & Sucrose / 0.2 & $\mathrm{NaNO} 3 / 0.013$ & 0.1 & $\begin{array}{l}\mathrm{KCl} / 0.003, \mathrm{MgC} 3 \mathrm{H} 9 \mathrm{O} 6 \mathrm{P} / 0.0003, \mathrm{FeSO} 4 / \\
0.00007, \mathrm{~K} 2 \mathrm{SO} 4 / 0.0023\end{array}$ \\
\hline Malt extract & 4.7 & Maltose / 0.087 & Vegetable peptone / 0.005 & 0.1 & Dextrin / 0.02 \\
\hline Plant extract & 7.1 & Whole barley extract & & 0.1 & \\
\hline Potato dextrose & 5.6 & Glucose / 0.3 & Potato extract / 0.027 & 0.1 & \\
\hline Sabouraud & 5.6 & Maltose / 0.27 & Mycological peptone $/ 0.067$ & 0.1 & \\
\hline
\end{tabular}




\subsection{Isolation of Endophytes}

For the first part of the experiment, the ability of the media to support the growth of ten pure cultures of endophytes was determined. The ten endophytes were derived from wild Irish populations of wall barley (Table 2). These had been isolated in a previous experiment and cultured on the half-strength malt extract medium specified in Table 1 and shown to have biocontrol (Murphy et al., 2014c) and biofertilisation (Murphy et al., 2015) properties for a barley cultivar (Propino). Single spores (i.e. only one spore) were used to inoculate the media and cultures were incubated for 28 days. The inoculants were prepared by removing $40 \mathrm{mg}$ of fungal material (conidia/spores and aerial mycelia) from the surface of each culture and then mixed (magnetically stirred) in $20 \mathrm{ml}$ pure water for $3 \mathrm{~min}$ at $25^{\circ} \mathrm{C}$ to ensure the inoculant was homogenised. For each medium, $250 \mu \mathrm{l}$ of the solution was inoculated onto the centre of $15 \times 900 \mathrm{~mm}$ culture dishes. All dishes were incubated in the dark at $25^{\circ} \mathrm{C}$ for 35 days. Plate coverage was calculated by measuring the radial growth of the fungus, and the time taken for the cultures to sporulate was recorded.

The second experiment determined the ability of the media to support the growth of endophytes emerging from root tissue. Twenty five culture dishes were prepared for each of the 7 media (corn meal agar (C), Czapek Dox agar (Z), malt extract agar (M), potato dextrose agar (P), Sabouraud agar (S), WPE 1:2 and WPE 1:30). Root pieces of approximately $10 \mathrm{~mm}$ in length were removed from the middle sections of healthy roots of wild wall barley from a population that had never previously produced any emergent endophytes on a malt extract agar medium. This population was chosen as we wanted to see if other media formulations would be more successful for endophyte recovery. Roots were surface-sterilised by washing soil residues from the roots using ultra-pure water, soaking in 5\% $\mathrm{NaClO}$ for $15 \mathrm{~min}$, then rinsing three times in ultra-pure water. 25 root pieces were placed onto each medium. A further 25 root pieces were placed onto the two WPE media but then flooded with $1 \mathrm{~mm}$ depth of full strength Bayer Phostrogen ${ }^{\circledR}$ All-Purpose Plant Food. All dishes were incubated at $25^{\circ} \mathrm{C}$ in the dark for 35 days.

Table 2. Nearest BLAST search matches for ITS sequences obtained from ten fungal root endophyte isolates derived from wild populations of Hordeum murinum ssp. murinum

\begin{tabular}{llcc}
\hline Isolate & Nearest BLAST match & \% Identical & Known properties* \\
\hline $040207(3)$ & Paecilomyces marquandii JQ013003 & 98.6 & $\mathrm{C}$ \\
040406(3) & Viridispora alata JF832678 & 94 & \\
$040605(2)$ & Cladosporium sp. GQ169491 & 99.6 & $\mathrm{TP}$ \\
040901(3) & Penicillium brevicompactum EU587331 & 94.8 & $\mathrm{THP}$ \\
040902(5) & Pyrenochaeta unguis-hominis JX966641 & 98 & $\mathrm{TH}$ \\
040906(4) & Uncultured Metarhizium KC797571 & 95.5 & $\mathrm{C}$ \\
040909(5) & Uncultured fungus FJ820798 & 93.8 & \\
$041004(5)$ & Exophiala oligosperma JN655630 & 100 & TH \\
041008(5) & Penicillium brevicompactum FJ884117 & 99.6 & THP \\
041010(3) & Penicillium sp. FR822844 & 99.4 & \\
\hline
\end{tabular}

*'Known properties' refers to whether the nearest named BLAST match has reported toxicity (T) for plants (P) and/or humans $(\mathrm{H})$, biofertilisation (F) or biocontrol (C) properties.

\subsection{Media Combinations}

Finally, combinations of commercial media and WPEs were tested. Each of the commercial culture media was combined with either the sterilized WPE 1:2 or a WPE 1:2 extract that was not sterilized and just vacuum filtered through a $0.2 \mu \mathrm{m}$ nylon mesh to exclude bacteria. The components were mixed with a medium:WPE ratio of 1 part medium to 30 parts WPE. As before, 25 root pieces were placed onto each mixture and the culture dishes incubated in the dark at $25^{\circ} \mathrm{C}$.

\subsection{Identification of endophytes}

Endophyte emergence was recorded over the next 35 days, and emergents were identified by morphological examination and by sequencing of the internal transcribed region (ITS) of nuclear ribosomal DNA (nrDNA). For the DNA analysis, $20 \mathrm{mg}$ of fungal material was scraped from the agar surface and placed into shaker tubes. DNA 
was extracted using a Qiagen DNeasy mini kit, following the Qiagen protocol, producing $200 \mu 1$ of DNA extracts for each isolate. PCR was carried out on the DNA extracts using the primers ITS1 and ITS4 (White et al., 1990). The thermal cycling parameters were programmed to optimise primer annealing, consisting of: 3 min at $95^{\circ} \mathrm{C} ; 9$ cycles of $1 \mathrm{~min}$ at $94^{\circ} \mathrm{C}, 1 \mathrm{~min}$ at $56^{\circ} \mathrm{C}, 2 \mathrm{~min}$ at $72^{\circ} \mathrm{C} ; 20$ cycles of $30 \mathrm{sec}$ at $94^{\circ} \mathrm{C}, 1 \mathrm{~min}$ at $56^{\circ} \mathrm{C}, 3 \mathrm{~min}$ at $72^{\circ} \mathrm{C}$, and a final extension for $7 \mathrm{~min}$ at $72^{\circ} \mathrm{C}$. PCR products were cleaned up using Exonuclease (New England Biolabs) and Shrimp Alkaline Phosphatase (ExoSAP; Roche). Purified PCR products underwent cycle sequencing using the forward ITS1 primer (4 pmol) or reverse ITS4 primer $(4 \mathrm{pmol})$ in separate reactions with the ABI BigDye 3.1 kit (Foster City, CA). The products were further purified using a BigDye XTerminator purification kit and protocol. DNA was sequenced using an Applied Biosystems 3130xL Genetic Analyzer. The isolate sequences were compared with GenBank accessions using the Basic Local Alignment Search Tool (BLAST).

Data analysis was carried out using ANOVA and Pearson's correlation statistical analyses supplied with the Data Analysis module within Microsoft Excel@ .

\section{Results}

\subsection{Endophyte Growth Tests}

For the simple growth tests using inoculants of existing endophyte isolates on each medium, Sabouraud gave the greatest mean radial growth of endophyte fungal mycelia after 35 days (Table 3). The proportion of plate cover with Sabouraud (calculated from the radial growth measurements), averaged over all isolates, was significantly greater than corn meal (ANOVA, $\mathrm{F}_{1,14}=5.80, \mathrm{P}<0.05$ ), Czapek Dox (ANOVA, $\mathrm{F}_{1,14}=5.90, \mathrm{P}<0.05$ ) and WPE media (ANOVA, $\mathrm{F}_{1,14}=21.89, \mathrm{P}<0.01$ ). The plate cover for the combined data for WPE media (WPE 1:2 plus WPE 1:30) was significantly less than all other media $(\mathrm{P}<0.01)$. Compared to the other media both WPE 1:2 and WPE 1:30 produced only very slow endophyte growth and the mycelial structure was generally very open, with clear hyphal branching and with growth spread right throughout the medium rather than concentrated on the agar surface as with the other media. Combined results for the two WPE media showed that only six of the ten endophytes produced visible mycelial growth: 040207(3), 040605(2), 040901(3), 040906(4), 041004(5), and 041008(5). Though they were very slow to develop, the first of these six endophytes eventually started to sporulate at 66 days, and all had produced spores at 78 days. The two media which produced the greatest proportion of plate cover, malt extract and Sabouraud, were also the only two media with maltose as the carbon source. Statistical analysis indicated no significant relationship between medium $\mathrm{pH}$ (Table 1) and mean plate cover of the endophytes.

The cultures growing on the WPE media took over five times longer to sporulate than all other media $(\mathrm{P}<0.001)$, and the Sabouraud medium induced significantly earlier sporulation than the corn meal medium (ANOVA, $\mathrm{F}_{1,14}=$ 9.45, $\mathrm{P}<0.01$ ) (Table 4).

Table 3. Mean radial growth \pm S.E. of isolates, represented as the proportion of culture dish covered after 28 days when inoculated with fungal endophyte isolates of Hordeum murinum ssp. murinum on one of six media

\begin{tabular}{ccccccc}
\hline \multicolumn{7}{c}{ Percentage plate cover on each medium } \\
\hline Isolate & Corn Meal & Czapek Dox & Malt Extract & Potato Dextrose & Sabouraud & WPE* \\
\hline $040207(3)$ & $20 \% \pm 2.8$ & $5 \% \pm 0.0$ & $10 \% \pm 0.0$ & $10 \% \pm 2.2$ & $10 \% \pm 0.0$ & $5 \% \pm 0.0$ \\
$040406(3)$ & $0.0 \%$ & $0.0 \%$ & $5 \% \pm 0.0$ & $0.0 \%$ & $10 \% \pm 2.0$ & $0.0 \%$ \\
$040605(2)$ & $30 \% \pm 3.9$ & $20 \% \pm 2.2$ & $25 \% \pm 2.4$ & $20 \% \pm 2.0$ & $20 \% \pm 2.2$ & $5 \% \pm 0.0$ \\
$040901(3)$ & $20 \% \pm 2.0$ & $0.0 \%$ & $35 \% \pm 4.3$ & $10 \% \pm 0.03$ & $35 \% \pm 6.1$ & $10 \% \pm 0.0$ \\
$040902(5)$ & $10 \% \pm 0.05$ & $30 \% \pm 3.9$ & $20 \% \pm 2.8$ & $20 \% \pm 3.2$ & $30 \% \pm 6.3$ & $0.0 \%$ \\
$040906(4)$ & $0.0 \%$ & $10 \% \pm 0.0$ & $20 \% \pm 2.2$ & $15 \% \pm 3.4$ & $15 \% \pm 3.9$ & $5 \% \pm 0.0$ \\
$040909(5)$ & $10 \% \pm 0.0$ & $25 \% \pm 4.3$ & $20 \% \pm 2.2$ & $20 \% \pm 5.2$ & $50 \% \pm 4.0$ & $0.0 \%$ \\
$041004(5)$ & $10 \% \pm 2.0$ & $10 \% \pm 2.0$ & $50 \% \pm 6.1$ & $30 \% \pm 3.9$ & $20 \% \pm 0.0$ & $10 \% \pm 0.0$ \\
$041008(5)$ & $10 \% \pm 0.05$ & $10 \% \pm 0.0$ & $10 \% \pm 0.5$ & $10 \% \pm 2.0$ & $20 \% \pm 2.4$ & $10 \% \pm 2.0$ \\
$041010(3)$ & $15 \% \pm 2.9$ & $10 \% \pm 0.0$ & $25 \% \pm 4.2$ & $20 \% \pm 2.6$ & $35 \% \pm 4.0$ & $0.0 \%$ \\
\hline Means & $\mathbf{1 2 . 5 \%}$ & $\mathbf{1 2 . 0 \%}$ & $\mathbf{2 2 . 0 \%}$ & $\mathbf{1 5 . 5 \%}$ & $\mathbf{2 4 . 5 \%}$ & $\mathbf{4 . 5 \%}$ \\
\hline
\end{tabular}

* The 'WPE' figures are for WPE 1:2 and WPE 1:30 combined. 
Table 4. Mean number of days to sporulation \pm S.E. from single spores of fungal endophyte isolates of Hordeum murinum ssp. murinum inoculated onto one of six media $(\mathrm{n}=15)$

\begin{tabular}{lcccccc}
\hline Isolate & Corn Meal & Czapek Dox & Malt Extract & Potato Dextrose & Sabouraud & WPE* \\
\hline $040207(3)$ & $28 \pm 0.3$ & $25 \pm 0.4$ & $23 \pm 0.3$ & $22 \pm 0.1$ & $20 \pm 0.2$ & $82 \pm 2.2$ \\
$040406(3)$ & $17 \pm 0.2$ & $14 \pm 0.2$ & $13 \pm 0.1$ & $12 \pm 0.0$ & $10 \pm 0.1$ & $76 \pm 0.4$ \\
$040605(2)$ & $14 \pm 0.2$ & $14 \pm 0.1$ & $12 \pm 0.2$ & $12 \pm 0.0$ & $10 \pm 0.0$ & $72 \pm 2.0$ \\
$040901(3)$ & $12 \pm 0.1$ & $9 \pm 0.0$ & $7 \pm 0.0$ & $10 \pm 0.0$ & $6 \pm 0.0$ & $48 \pm 2.0$ \\
$040902(5)$ & $15 \pm 0.1$ & $11 \pm 0.0$ & $12 \pm 0.2$ & $10 \pm 0.1$ & $9 \pm 0.0$ & $60 \pm 0.5$ \\
$040906(4)$ & $16 \pm 0.2$ & $12 \pm 0.1$ & $12 \pm 0.3$ & $11 \pm 0.1$ & $9 \pm 0.1$ & $65 \pm 0.4$ \\
$040909(5)$ & $14 \pm 0.2$ & $10 \pm 0.2$ & $10 \pm 0.0$ & $10 \pm 0.0$ & $8 \pm 0.0$ & $72 \pm 2.0$ \\
$041004(5)$ & $12 \pm 0.1$ & $9 \pm 0.0$ & $7 \pm 0.0$ & $7 \pm 0.0$ & $7 \pm 0.1$ & $51 \pm 0.5$ \\
$041008(5)$ & $12 \pm 0.1$ & $11 \pm 0.1$ & $8 \pm 0.0$ & $9 \pm 0.1$ & $8 \pm 0.0$ & $72 \pm 2.0$ \\
$041010(3)$ & $14 \pm 0.2$ & $10 \pm 0.0$ & $10 \pm 0.1$ & $9 \pm 0.1$ & $7 \pm 0.0$ & $61 \pm 0.4$ \\
\hline Means & $\mathbf{1 5}$ & $\mathbf{1 3}$ & $\mathbf{1 1}$ & $\mathbf{1 1}$ & $\mathbf{9}$ & $\mathbf{6 6}$ \\
\hline
\end{tabular}

*The 'WPE' figures are for WPE 1:2 and WPE 1:30 combined.

\subsection{Endophyte Recovery}

The total number of emergent endophytes (morphotypes) from root pieces varied widely depending on the different media: corn meal and Sabouraud both supported a total of ten endophyte emergents, WPE 1:2 supported eight, potato dextrose supported five, Czapek Dox supported four and the remaining WPE media (WPE 1:30 and flooded WPE plates) and malt extract supported none (Table 5). The number of different endophyte emergents on each media was less varied: four of the media - corn meal, potato dextrose, Sabouraud and WPE 1:2 - supported three different endophytes, while Czapek Dox had two. The malt extract medium had no emergent endophytes, confirming previous results (see Materials and Methods).

Table 5. Number and identity of endophyte emergents (morphotypes) from roots of Hordeum murinum ssp. murinum after 35 days $(\mathrm{n}=25)$

\begin{tabular}{lccl}
\hline \multicolumn{4}{c}{$\begin{array}{c}\text { Number of endophyte emergents on each } \\
\text { medium }\end{array}$} \\
\hline Medium & Total emergents & Different emergents & Fungal genera identified \\
\hline Corn Meal & 10 & 3 & Cladosporium, Fusarium, Penicillium \\
Czapek Dox & 4 & 2 & Fusarium, Metarhizium \\
Malt Extract & 0 & 0 & \\
Potato Dextrose & 5 & 3 & Cladosporium, 2 x Penicillium \\
Sabouraud & 10 & 3 & Paecilomyces, 2 x Penicillium \\
WPE 1:2 + Agar & 8 & 3 & Exophiala, 2 x Penicillium \\
WPE 1:30 + Agar & 0 & 0 & \\
WPE 1:30 + Agar, & 0 & 0 & \\
flooded & 4.6 & 1.8 & \\
Means & & & \\
\hline
\end{tabular}

\subsection{Media Combinations}

When the standard commercial media were combined with WPE 1:2 in a medium: WPE ratio of 1:30, we found that there were also some significant differences in endophyte emergents between media (Table 6). Both the malt extract: WPE and potato dextrose:WPE mixtures had a significantly greater number of emergents $(\mathrm{P}<0.01)$ than 
the other media mixtures, while the malt extract:WPE mixture also had a significantly greater number of different emergents (ANOVA series, $\mathrm{P}<0.05$ ) than all of the other mixtures. The mean proportion of culture plate covered by the fungi ranged from $10 \%$ for the Sabouraud:WPE mix to $100 \%$ for the Czapek Dox:WPE mix. The fully sterilized media:WPE mixtures had a significantly greater number of total endophyte emergents than the simply filtered mixtures (ANOVA, $\mathrm{F}_{1,70}=50.7, \mathrm{P}<0.01$ ) but demonstrated no difference in mean proportion of plate cover.

\subsection{Endophyte Identities}

When the ITS sequences obtained from the emergent endophytes recovered from the root pieces were compared with GenBank accessions, it was found that the isolates were most closely matched in pairwise similarity to six different genera - Cladosporium, Exophiala, Fusarium, Metarhizium, Paecilomyces and Penicillium (Table 5). These sequences were deposited in GenBank (accession numbers: KP309885, KP309892, KP309900, KP309918, KP309934, KP309936, KP309937, KP309941, KP309943, KP309944, KP309981, KP309985, KP309986).

Table 6. Number of endophyte emergents from roots of Hordeum murinum ssp. murinum after 35 days $(\mathrm{n}=25)$

\begin{tabular}{lcccc}
\hline Medium & $\begin{array}{c}\text { Total emergents } \\
\text { Autoclv/Filtered* }\end{array}$ & $\begin{array}{c}\text { Different } \\
\text { emergents } \\
\text { Autoclv/Filtered* }\end{array}$ & $\begin{array}{c}\text { \% plate cover } \\
\text { Autoclv/Filtered* }\end{array}$ & $\begin{array}{c}\text { Mean \% plate } \\
\text { cover }\end{array}$ \\
\hline Corn Meal:WPE & $3 / 2$ & $1 / 1$ & $33 / 33$ & 33 \\
Czapek Dox:WPE & $2 / 3$ & $1 / 2$ & $100 / 100$ & 100 \\
Malt Extract:WPE & $12 / 3$ & $6 / 1$ & $75 / 50$ & 62.5 \\
Potato & $17 / 4$ & $3 / 2$ & $100 / 50$ & 75 \\
Dextrose:WPE & $1 / 1$ & $1 / 1$ & $10 / 10$ & 10 \\
Sabouraud:WPE & $7 / 2.6$ & $2.4 / 1.4$ & $64 / 49$ & 56 \\
\hline Means & & & & \\
\hline
\end{tabular}

*'Autoclv / Filtered' refers to whether medium mix has been either unfiltered and autoclaved or just filtered through a $0.2 \mu \mathrm{m}$ mesh. Fungal genera identified for the emergents: Cladosporium, Exophiala, Fusarium, Paecilomyces and Penicillium.

\section{Discussion}

We have found that the use of different agar-based cultural media results in significantly varied responses in a set of ten culturable fungal root endophytes isolated from populations of wall barley (Hordeum murinum ssp. murinum L). Perhaps the most surprising result was the poor initial performance of the medium based solely on various concentrations of whole plant extract (WPE), which gave significantly less radial extension, and slower growth and sporulation. However, over the long term the endophyte cultures in the WPE media had a much more well defined mycelial growth character than that observed for the other media, with an almost fractal pattern. While our results may indicate that WPE can inhibit the growth and development of these particular endophytes, previous studies have shown contradictory effects. Most of these studies support our finding that WPE has significant anti-fungal activity in vitro (Mohana \& Raveesha, 2007; Suleiman \& Ogduna, 2010; Dellavalle et al., 2011; Minz, 2012; Yenn et al., 2012). This is in contrast to other studies which have reported that WPE acted as growth promoters for endophytic fungi and bacteria (Nour et al., 2012; Osman et al., 2013).

A WPE plus malt extract or potato dextrose medium gave the greatest number of total endophyte emergents, while only the plant juice plus malt extract mixture had a significantly greater number of different endophyte emergents. As both the malt extract medium and the pure WPE media had no emergents when used alone, it seems that it is the particular combination of medium components that is important for the initial isolation of fungal root endophytes. These results, combined with the fact that the Phostrogen flooded plant juice media produced no endophyte emergents, suggest that a greater range of fungal root endophytes emerge preferentially onto a semi-solid nutrient-enriched substrate containing plant extract.

The reasons why the autoclaved plant juice based media had significantly more endophyte emergents than the simply filtered plant juice based media are not entirely clear - in fact they are probably counterintuitive. While the mesh filter excluded all bacteria, the resultant filtrate would contain most of the small plant-derived molecules. 
Though these molecules may contribute to producing a medium that would be more similar to the plant apoplastic environment than the autoclaved media, they may not necessarily encourage more endophyte emergence, due to the inhibitory effects of WPE on some fungi (as discussed earlier). Viruses would be small enough to pass through the filter (Sanual \& Van Regenmortel, 1995), and endophytes which would emerge from plant roots onto the culture medium as filamentous fungi are known to host many mycoviruses (Pearson, Beever, Boine, \& Arthur, 2009), which can have various effects on the fungus. Some types of mycovirus have been shown to be detrimental to the host and reduce fungal growth (Van Diepeningen et al., 2006). Autoclaving the media would destroy any mycoviruses but they would still be present in the simply filtered medium, and their presence may have reduced endophyte emergence (Herrero Asensio et al., 2013).

The growth of individual isolates (as measured by proportion of culture plate cover) varied significantly between media. The two media which produced the greatest radial growth, malt extract and Sabouraud, were also the only two media with maltose as the carbon source. Maltose is derived from barley so may have been a more accessible and useable carbon source for these endophytes from a wild barley relative. However the radial growth and proportion of plate cover between isolates, ranging from $10 \%$ to $100 \%$, is not a reliable indicator of the rate of fungal growth but is a function of endophyte identity.

Sporulation timing and quantity is also a function of endophyte identity, so comparisons between isolates would be meaningless; but we did find that each of the endophyte isolates on the pure WPE media took significantly longer to sporulate than on all the other media. This would seem to indicate that the endophytes require higher nutrient availability than exists in plant tissue, as well as other environmental cues, to sporulate early. Most fungal endophytes do not sporulate within plant tissue, and the presence of WPE in the media may have had an inhibitory effect on sporulation.

The statistical analyses indicated no significant relationship between medium $\mathrm{pH}$ and proportion of plate covered by the fungi, so these particular endophytes which were recovered from a site with neutral soil pH (7.2) may have no particular preference for this soil character. The roots were intentionally sampled from wall barley plants in the same population, so that results for every cultural treatment would be comparable, without the confounding environmental factors resulting from multiple sampling sites.

The results may also indicate which endophyte is more active inside barley tissue, as a WPE medium based on host extract is the closest medium to the normal endophyte environment. However, this possibility is yet to be rigorously tested by methods such as quantitative PCR. The isolates which had the greatest radial growth in our study have also been shown to be effective suppressants of seed-borne fungal infections of barley (Murphy et al., 2014c) as well as inducing yield and biomass increases (Murphy et al., 2015).

Overall, our results indicate that different media or medium combinations may be suitable for different purposes when culturing the fungal root endophytes we have isolated from wall barley. A medium that produces optimal fungal growth or early sporulation may not be the most suitable for initial recovery of endophytes from roots. If retarding endophyte growth and sporulation is the purpose then a WPE based medium may be more suitable than media richer in nutrients. An extra benefit associated with the use of a WPE based medium is the slow but fine structure of mycelial growth, increasing the visual clarity of fungal components.

These results may have relevance for the mass production of beneficial endophytes for agricultural use, as the correct balance of medium components is crucial in developing economically viable biotechnological products based on endophytes. By optimising the cultural conditions to suit endophyte emergence, there is potential for extracting a greater range of endophytes from plant tissue, perhaps even previously unculturable endophytes. Finally, tailoring the culture medium by incorporating WPE may remove the need for continuously 'refreshing' the endophytes in planta in order to retain efficacy, thus removing one of the major hurdles to maintaining competent strains over the long-term.

\section{Acknowledgements}

We thank: Helena Murphy for proof reading and the de-cluttering of technical terms; laboratory technicians at Trinity College Dublin for providing supplies and technical support; Nabil Hegazi of the University of Cairo for initial discussion and inspiration regarding the use of plant based media. Trinity College Dublin provided financial support through a PhD studentship grant.

\section{References}

Achatz, B., Rüden, S., Andrade, D., Neumann, E., Pons-Kühnemann, J., Kogel, K.-H., Waller, F. (2010). Root colonization by Piriformospora indica enhances grain yield in barley under diverse nutrient regimes by 
accelerating plant development. Plant and Soil, 333(1-2), 59-70. http://dx.doi.org/10.1007/s11104 -010-0319-0

Behie, S. W., \& Bidochka, M. J. (2013). Potential agricultural benefits through biotechnological manipulation of plant fungal associations. BioEssays : News and Reviews in Molecular, Cellular and Developmental Biology, 35(4), 328-31. http://dx.doi.org/10.1002/bies.201200147

Dellavalle, P.D., Cabrera, A., Alem, D., Larrañaga, P., Ferreira, F., \& Rizza, M.D. (2011). Antifungal activity of medicinal plants extracts against phytopathogenic fungus Alternaria spp. Chilean Journal of Agricultural Research, 71, 231-239. http://dx.doi.org/ 10.1007/s11418-007-0216-x

Herrero Asensio, N., Sánchez Márquez, S., \& Zabalgogeazcoa, I. (2013). Mycovirus effect on the endophytic establishment of the entomopathogenic fungus Tolypocladium cylindrosporum in tomato and bean plants. BioControl, 58, 225-232. http://dx.doi.org/ 10.1007/s10526-012-9476-9

Knob, A., Beitel, S. M., Fortkamp, D., Terrasan, C. R. F., \& de Almeida, A. F. (2013). Production, Purification, and Characterization of a Major Penicillium glabrum Xylanase Using Brewer's Spent Grain as Substrate. BioMedical Research International, (728735), 1-8. http://dx.doi.org/ 10.1155/2013/728735

Kumar, V., Sahai, V., \& Bisaria, V. S. (2011). High-density spore production of Piriformospora indica, a plant growth-promoting endophyte, by optimization of nutritional and cultural parameters. Bioresearch Technology, 102, 3169-75. http://dx.doi.org/ 10.1016/j.biortech.2010.10.116

Kusari, S., Singh, S., \& Jayabaskaran, C. (2014). Biotechnological potential of plant-associated endophytic fungi: hope versus hype. Trends in Biotechnology, 32(6), 297-303. http://dx.doi.org/10.1016/j.tibtech.2014.03.009

Meletiadis, J., Meis, J. F. G. M., Mouton, J. W., \& Verweij, P. E. (2001). Analysis of Growth Characteristics of Filamentous Fungi in Different Nutrient Media. Journal of Clinical Microbiology, 39(2), 478-484. http://dx.doi.org/10.1128/JCM.39.2.478

Minz, S. (2012). The Effect of Plant Extracts on the Growth of Wilt Causing Fungi Fusarium oxysporum. Journal of Pharmaceutical Biology Sciences, 4, 13-16.

Mohana, D. C., \& Raveesha, K. (2007). Anti-fungal evaluation of some plant extracts against some plant pathogenic field and storage fungi. Journal of Agricultural Technology, 4, 119-137.

Murphy, B. R., Doohan, F. M., \& Hodkinson, T. R. (2014). Fungal endophytes of barley roots. The Journal of Agricultural Science, 152, 602-615. http://dx.doi.org/10.1017/S0021859613000348

Murphy, B. R., Doohan, F. M., \& Hodkinson, T. R. (2014b). Yield increase induced by the fungal root endophyte Piriformospora indica in barley grown at low temperature is nutrient limited. Symbiosis. http://dx.doi.org/10.1007/s13199-014-0268-0

Murphy, B. R., Doohan, F. M., \& Hodkinson, T. R. (2014c). Persistent fungal root endophytes isolated from a wild barley species suppress seed- borne infections in a barley cultivar. Biocontrol, 60, 281-292. http://dx.doi.org/ 10.1007/s10526-014-9642-3

Murphy, B. R., Doohan, F. M., \& Hodkinson, T. R. (2015). Fungal root endophytes of a wild barley species increase yield in a nutrient-stressed barley cultivar. Symbiosis, 65, 1-7. http://dx.doi.org/10.1007/ s13199-015-0314-6

Newton, A. C., Flavell, A. J., George, T. S., Leat, P., Mullholland, B., Ramsay, L., ... Swanston, J. S. (2011). Crops that feed the world 4. Barley: a resilient crop? Strengths and weaknesses in the context of food security. Food Security, 3, 141-178. http://dx.doi.org/ 10.1007/s12571-011-0126-3

Nour, E. H., Hamza, M. A., Fayez, M., Monib, M., Ruppel, S., \& Hegazi, N. A. (2012). The crude plant juices of desert plants as appropriate culture media for the cultivation of rhizospheric microorganisms. Journal of Advanced Research, 3(1), 35-43. http://dx.doi.org/10.1016/j.jare.2011.03.002

Osman, Z. A., Elsanousi, S. M., \& Elsheikh, E. A. E. (2013). Plant materials as probable growth promoters for certain fungi. Asian Journal of Agricultural Science, 3, 87-93.

Pearson, M. N., Beever, R. E., Boine, B., \& Arthur, K. (2009). Mycoviruses of filamentous fungi and their relevance to plant pathology. Molecular Plant Pathology, 10(1), 115-28. http://dx.doi.org/10.1111/j.1 364-3703.2008.00503.x 
Sanual, H., \& Van Regenmortel, M. H. V. (1995). Mapping of viral conformational epitopes using biosensor measurements. Journal of Immunological Methods, 183, 33-41. http://dx.doi.org/10.1016/0022-1759(95) $00021-2$

Singh, A. A., Kumari, M., Rai, M. K., \& Varma, A. (2003). Biotechnological Importance of Piriformospora indica Verma et al-A Novel Symbiotic Mycorrhiza-like Fungus : An Overview. Indian Journal of Biotechnology, 2(January), 65-75.

Stein, E., Molitor, A., Kogel, K.-H., \& Waller, F. (2008). Systemic resistance in Arabidopsis conferred by the mycorrhizal fungus Piriformospora indica requires jasmonic acid signaling and the cytoplasmic function of NPR1. Plant \& Cell Physiology, 49(11), 1747-51. http://dx.doi.org/10.1093/pcp/pcn147

Suleiman, M. N., \& Ogundana, A.F. (2010). Evaluation of two plant leaf extracts on fungi associated with biodeterioration of cashew nuts in storage. Annals of Biological Research, 1, 41-44.

Van Diepeningen, A.D., Debets, A.J.M., \& Hoekstra, R.F. (2006). Dynamics of dsRNA mycoviruses in black Aspergillus populations. Fungal Genetics and Biolology, 43, 446-452. http://dx.doi.org/10.1016/j.fgb. 2006.01.014

Waller, F., Achatz, B., Baltruschat, H., Fodor, J., Becker, K., Fischer, M., \& Kogel, K.-H. (2005). The endophytic fungus Piriformospora indica reprograms barley to salt-stress tolerance, disease resistance, and higher yield. Proceedings of the National Academy of Sciences of the United States of America, 102(38), 13386-91. http://dx.doi.org/10.1073/pnas.0504423102

Zadoks, J. C., Chang, T. T., \& Konzak, C. F. (1974). A Decimal Code for the Growth Stages of Cereals. Weed Research, 14, 415-421.

\section{Copyrights}

Copyright for this article is retained by the author(s), with first publication rights granted to the journal.

This is an open-access article distributed under the terms and conditions of the Creative Commons Attribution license (http://creativecommons.org/licenses/by/3.0/). 